Notes sur les métaphores fondatrices de la connaissance sociologique

\title{
Giovanni Busino
}

\section{(2) OpenEdition}

\section{Journals}

Édition électronique

URL : http://journals.openedition.org/ress/711

DOI : $10.4000 /$ ress. 711

ISSN : 1663-4446

Éditeur

Librairie Droz

\section{Édition imprimée}

Date de publication : 1 février 2000

Pagination : 69-81

ISBN : 2-600-00409-2

ISSN : 0048-8046

\section{Référence électronique}

Giovanni Busino, « Notes sur les métaphores fondatrices de la connaissance sociologique », Revue européenne des sciences sociales [En ligne], XXXVIII-117| 2000, mis en ligne le 17 décembre 2009, consulté le 19 avril 2019. URL : http://journals.openedition.org/ress/711 ; DOI : 10.4000/ress.711 


\section{NOTES SUR LES MÉTAPHORES FONDATRICES DE LA CONNAISSANCE SOCIOLOGIQUE}

La publication du livre Impostures intellectuelles de Sokal et Bricmont a relancé récemment le débat sur l'importance et le rôle des métaphores dans l'élaboration des connaissances dans les sciences de l'homme et de la société. Bien que ces deux auteurs aient clairement écrit de ne point sous-évaluer la dimension heuristique des métaphores, d'en contester uniquement les usages abusifs et incontrôlés, surtout ceux dépourvus de justifications empiriques ou conceptuelles et quand ces mêmes usages génèrent des véritables mystifications ${ }^{1},-$ néanmoins nombreux sont ceux qui persistent à les accuser d'être obsédés par une soi-disant «positivité » de la science, notamment de la physique, d'ignorer «que les sciences humaines et sociales sont d'un ordre de difficulté sans commune mesure avec l'étude de la nature, que leur textualité est d'une toute autre nature», qu'on ne peut juger ces sciences qu' «en fonction de leurs critères propres de validité », sans quoi par ailleurs il serait impossible «de faire émerger des effets de sens bien plus subtils que le plat accord entre une expérimentation et une théorisation toutes deux technicisées et instrumentalisées $»^{2}$.

Jacques Bouveresse, dans un pamphlet à la fois caustique et narquois mais très revigorant ${ }^{3}$, s'est élevé contre une telle conception, contre les emprunts abusifs à d'autres domaines, contre les constructions théoriques faisant l'économie des données empiriques spécifiques, et il a montré, à coups de griffes acérées, les effets pervers produits lorsque les métaphores sont utilisées sans contrôle. Les emprunts abusifs de concepts ou de théorèmes ne donnent jamais la dignité de science aux énoncés sans contenus ni même de la solidité factice aux arguments qui disent tout de rien ou rien du tout. Au contraire, ils provoquent de la confusion, du verbiage, des amphigouris.

Ces controverses, parfois brouillonnes et déconcertantes ${ }^{4}$, ont eu au moins le mérite de faire ouvrir à nouveau le dossier, au demeurant déjà fort épais ${ }^{5}$, sur l'im-

A. Sokal \& J. Bricmont, Impostures intellectuelles, Paris, Le livre de poche/Biblio Essais, 1999, $2^{e}$ éd., p. 15 note 4 et p. 22.

2 J.-M. Lévy-Leblond, La méprise et le mépris, in: Sous la direction de B. Jurdant, Impostures scientifiques. Les malentendus de l'affaire Sokal, Paris, La Découverte/Alliage, 1998, pp. 27-42.

3 J. Bouveresse, Prodiges et vertiges de l'analogie. De l'abus des belles-lettres dans la pensée, Paris, Raisons d'agir Editions, 1999.

4 J'en ai fait l'historique ainsi qu'une analyse critique dans Il dibattito sulla scienza nelle ricerche recenti. Dal «Programma forte» alle controversie dell" "Affare Sokal», in «Rivista storica italiana», CXLI, settembre 1999, n. 3, pp. 707-756.

5 La bibliographie sur le sujet est immense. Pour une première orientation, cf. M. Black, Models and Metaphors. Studies in Language and Philosophy, Ithaca, Cornell University Press, 1962; 
portance et la fécondité des analogies et des métaphores pour le travail cognitif des disciplines non expérimentales, celles qui ne peuvent ni ne savent construire leurs objets comme des objets formels. En même temps, ces mêmes controverses nous ont rappelé que les schèmes analogiques et métaphoriques constituent invariablement les bases des catégories, des présupposés, des concepts et des schèmes d'énonciation des sciences de l'homme et de la société, qu'ils permettent de construire une structure du réel par le biais de la ressemblance des rapports entre des réalités hétérogènes et des isotopies différentes, moyennant l'extension du sens antérieur d'un signifiant, l'attribution de deux sens un peu différents d'un même mot (l'antanaclase), l'intégration de perspectives variées, le gommage de tout ce que pourrait révéler que les variables et les structures construites ne sont pas comparables entre elles sous tous les rapports. C'est ainsi que les chercheurs arrivent à extraire du sens d'un contexte et à l'introduire dans un autre, à croiser des cadres de références hétérogènes et à rendre intelligibles des concepts obscurs et indistincts en les raccordant à des concepts clairs et distincts.

En un mot, les schèmes analogiques et métaphoriques permettent aux chercheurs, parfois après une épuration sommaire de certains contenus intuitifs, d'étudier les processus sociaux et de construire des représentations plausibles du monde social. En outre, ces schèmes leur permettent de généraliser les descriptions de phénomènes qui échappent aux transcriptions directes, d'aller au-delà des énumérations des caractères des phénomènes observables, de circonscrire des nouveaux champs perceptifs, d'élaborer des nouveaux langages et des nouvelles approches dans le but de bien poser les problèmes et d'en trouver, le cas échéant, des solutions. Toutefois, en dépit de tout, l'épistémologie des sciences sociales n'offre aux chercheurs aucune véritable théorie systématique susceptible de leurs faire distinguer les bonnes des mauvaises métaphores, les modèles des métaphores, les analogies de proportion de celles d'attribution. A cause de cela, en outre, les risques d'amalgames sont nombreux, courants, inévitables, tandis que le verbiage pur et simple qui mêle les effets avec les faits est à l'origine de maintes confusions, d'ambiguïtés et de multiples paradoxes.

Une histoire des usages et du modus operandi des métaphores, mais aussi des synecdoques, des métonymies et des tropes les plus courants, pourrait permettre d'expliquer les raisons de ces carences et peut-être de mettre en évidence de quelle façon la structuration de l'analyse sociologique, même lorsqu'elle fait appel aux modèles analogiques et aux modèles iconiques, aux schèmes analogiques, aux typologies ${ }^{6}$, reste toujours le produit d'intuitions et d'utilisations métaphoriques.

W.A.Shibles, An Analysis of the Metaphor in the Light of W. M. Urban's Theories, The HagueParis, Mouton, 1971; Id., Metaphor. An Annoted Bibliography and History, Whiterwater, Wis., Language Press, 1971; Id., Essays on Metaphor, Whitenwater, Wis., Language Press, 1972; Id., Metaphor. A Bibliography of post-1970, Amsterdam, J. Benjamins, 1985. Importants et stimulants demeurent les travaux de P. Ricœur, La métaphore vive, Paris, Seuil, 1975; S. R. Levin, The Semantics of Metaphor, Baltimore, Johns Hopkins University, 1977; A. Henry, Métonymie et métaphore, Bruxelles, Académie royale de Belgique, 1984; E. Montuschi, Le metafore scientifiche, Milano, Angeli, 1993; M. Hesse, The Cognitive Claims of Metaphor, in «Journal of Speculative Philosophy», vol. 2, 1988, n. 1, pp. 1-16; J. Schlanger, Connaissance et métaphore, in «Revue de synthèse », $4^{\text {e }}$ S., n. 4, oct.-déc. 1995, pp. 579-592.

6 Récemment D. Schnapper a publié La compréhension sociologique. Démarche de l'analyse typologique, Paris, PUF, 1999, où sont fort bien présentés ces modèles conceptuels lesquels, pour 
En effet, contrairement aux affirmations d'Aristote, pour lequel la métaphore délocalise «un mot vers un sens différent de son sens originel» et donne «à un objet un nom qui désigne un autre objet ${ }^{7}$, les sociologues donnent pour acquis qu'elle transfère non seulement les termes mais également des systèmes conceptuels, qu'elle favorise les échanges d'un niveau ou degré à d'autres niveaux ou degrés, qu'elle assure l'élaboration d'approches analytiques stéréoscopiques et qu'elle est toujours porteuse de sens, notamment quand il n'y a pas de critères certains de proportion et de correspondance et que les «objets » n'existent pas indépendamment des conceptions théoriques avec lesquelles ils ont été construits. En vérité, si la métaphore permet de former de nouveaux prédicats d'observation, de regarder les phénomènes sociaux «comme si» et de les rendre intelligibles en tant qu'objets particuliers d'expérience en les dotant de buts, de fonctions, d'objectifs spécifiques, néanmoins elle privilégie un élément au détriment de tous les autres, fait ressortir une ressemblance mais dissimule les différences ou les ramène à l'identité.

Le rattachement ou/et le rapprochement d'éléments hétérogènes impliquent forcément la formation d'une longue chaîne des métaphores. A ce propos, l'organicisme offre des exemples significatifs. Les métaphores biologiques pour l'interprétation des phénomènes sociaux ont été utilisées dès l'aube de la sociologie. Pour rendre intelligibles les phénomènes sociaux, de Comte à Spencer, on a fait appel aux figures de l'organisme biologique et puis on les a détaillées avec les métaphores de la sélection, de l'hérédité, de la lutte pour l'existence, des facteurs bio-sociaux et démographiques. La sociobiologie, pour rendre intelligibles les comportements humains, fait des larges emprunts à l'évolution, à l'éthologie, à la génétique des populations, à la théorie des comportements sociaux des animaux.

Un petit échantillon de ces usages, au demeurant nombreux et variés, devrait permettre d'évaluer ici, tout au moins en première approximation, l'importance cognitive des métaphores pour la construction des objets d'expérience, des représentations sociales, pour la compréhension des pratiques des sociologues (comment manipulons-nous les similarités, les similés, les identités, les différences?) et du langage de la sociologie (comment arrive-t-elle à intégrer les points de vue et les multiples perspectives sans corroder les différences spécifiques des phénomènes étudiés? $)^{8}$.

dégager de l'intelligibilité, doivent construire les traits essentiels d'une individualité historique, d'une institution (le socialisme, le capitalisme, le protestantisme, la société industrielle, l'Etatprovidence, la citoyenneté, etc.), d'une relation sociale (l'identité religieuse) ou d'une expérience vécue (le chômage, la pauvreté). Cette étude démontre que les typologies permettent d'analyser les liens entre les conduites des individus, le sens qu'ils leur donnent, et les caractéristiques de l'«individualité historique» de la société.

7 Aristote, Poétique, trad. J.Hardy, Paris, Les Belles-Lettres, 1965, XXI, et Réthorique, trad. M. Dufour, Paris, Les Belles-Lettres, 1967, III : x.

8 Ici nous ne pouvons pas aborder la question très controversée des contenus (émotionnels, cognitifs, objectifs, subjectifs), des méthodes (quantitatives, qualitatives, explication causale, explication herméneutique) de l'histoire de la sociologie tels qu'ils ont été spécifiés par P. Lazarsfeld in Notes on the History of Quantification in Sociology: Trends, Sources and Problems, «Isis », vol. 52, $2^{\mathrm{e}}$ partie, juin 1961, pp. 277-333 (en français dans le volume du même Philosophie des sciences sociales, Paris, Gallimard, 1970, pp. 75-184), par W. Lepenies, Die drei Kulturen : Soziologie zwischen Literatur und Wissenschaft, München, Hanser, 1985 (trad. française: Les trois 
Les sociologues supposent que les propriétés stéréoscopiques des métaphores transmettent des connaissances utiles pour leurs recherches, bien que leur nature soit disparate. En effet, certaines de ces métaphores aident à élaborer un signe qui possède les propriétés du signe visuel représentatif. Les logiciens les nomment «métaphores iconiques». Porteuses de propositions synthétiques, analytiques et/ou logiques, ces métaphores font référence à elles-mêmes au sein d'un même système ou encore aux jeux des interrelations entre une catégorie quelconque et l'un ou l'autre des spécimens pris en considération. Elles aident à décrire les phénomènes et beaucoup moins à en comparer les ressemblances et les différences. Un exemple paradigmatique nous est donné par le sociologue Vilfredo Pareto quand il utilise la métaphore de la toupie renversée ou de la flèche pointue pour étaler sa théorie de la distribution de la richesse dans les sociétés du passé et du présent, ou encore quand il fait appel à la métaphore géologique pour élaborer la théorie de la circulation, ascendante et descendante, des élites entre les différentes couches et strates de la société. D'autres exemples nous sont donnés par les types idéaux utilisés par Max Weber dans ses études sur les religions mondiales et par Emile Durkheim dans ses livres sur la division du travail social et sur le suicide.

D'une autre nature sont les «métaphores analogiques», lesquelles impliquent un système des relations, entre des espèces et des catégories, fondé sur l'égalité des rapports; elles créent des images par comparaison et/ou par contraste et laissent entrevoir à quoi ces images ressemblent. L'épidémiologie, par exemple, a permis à certains sociologues d'analyser la propagation, la fréquence, la distribution et l'évolution des rumeurs sociales, et l'oncologie à élaborer la doctrine du racisme en tant que cancer moulinant le tissu social. Les «métaphores analogiques » favorisent la formulation de nouvelles théories et d'autres représentations du réel grâce à l'assomption de certaines similitudes entre différents rapports. Le raisonnement par induction, qui passe, à la suite d'inférences successives, du particulier au général, est la forme de procédure analogique la plus usitée en sciences sociales.

Les «métaphores illustratives» permettent, quant à elles, de faire appel à un simile, à un comme si. Une telle procédure, certes utile du point de vue analytique, dispense de la comparaison à dimensions multiples et dissimule spécieusement ce qu'elle est supposée illustrer. Gordon Tullock, et d'autres avec lui, utilise ce genre de métaphores illustratives lorsqu'il applique à la politique et à la bureaucratie la théorie du marché (monopole, concurrence, coût d'information, etc.), à l'électeur la théorie du consommateur et métamorphose la rationalité économique en rationalité politique et les intérêts individuels en intérêts généraux.

En sociologie, les métaphores de base les plus couramment utilisées prétendent décrire des univers déterminés et revendiquent la possession de contenus spécifiques. La théorie de l'habitus et celle des champs de Pierre Bourdieu en sont des exemples. Ces métaphores de base, constituant l'ontologie implicite de la sociologie, sont: (1) le mécanicisme (traiter les phénomènes sociaux comme des variétés des phénomènes physiques); (2) l'organicisme (rendre intelligibles les

cultures. Entre science et littérature l'avènement de la sociologie, Paris, Ed. de la Maison des sciences de l'homme, 1990, par R. Boudon, Comment écrire l' histoire des sciences sociales?, in «Communications », n. 54, 1992, pp. 299-317, et par L. Quéré, La sociologie à l'épreuve de l'herméneutique. Essais d'épistémologie des sciences sociales, Paris, L'Harmattan, 1999. 
phénomènes sociaux en s'appuyant sur les sciences de l'organisation du vivant); (3) l'économisme (concevoir l'acteur social comme capable d'intentions, de stratégies, de calcul, de rationalité instrumentale et procédurale); (4) le sociologisme (prééminence de la totalité sur les parties la constituant, la société surplombe les individus); (5) le langage (l'action réciproque des êtres humains et les signes qui la rendent visible n'est possible que par l'appartenance à une communauté de significations d'où la primauté des signes langagiers); (6) la mise en scène théâtrale, la dramaturgie (les comportements sont régis par le cadre définissant la situation, d'où les emprunts aux rituels de présentation et d'interaction du théâtre); (7) le jeu (dans les situations d'interaction de caractère stratégique l'agent est un joueur qui vise à maximiser ses avantages); (8) le réseau (tantôt c'est le refus de la dichotomie nature/société, science/politique, sujet/objet, homme/animal, etc., la totalité devient une structure contraignante, constituée d'éléments liés par des degrés d'interdépendance et faisant émerger des relations et des interactions, tantôt c'est le paradigme des technologies de l'information qui offre les bases matérielles de son extension à la structure sociale tout entière).

L'application que les sociologues font de ces huit métaphores de base (mais il y en a d'autres moins courantes) n'est pas uniforme. Elle varie d'un auteur à l'autre et rarement il est possible de déduire les conditions à venir des conditions observées.

Prenons le cas de la métaphore tirée de (1) la mécanique ou de la thermodynamique . Pareto y fait constamment référence. La théorie de l'équilibre est au centre de toutes ses analyses. La métaphore de l'équilibre (construite sur l'idée d'égalité de force entre deux ou plusieurs réalités opposées et sur l'idée de repos découlant pour ceux qui s'y trouvent soumis) ainsi que les notions de stabilité, instabilité et équilibre dynamique, lui servent pour tenir ensemble et puis analyser les phénomènes économiques et sociologiques. Talcott Parsons, lui aussi, fonde sa théorie du système social sur la notion d'équilibre. Mais ni Pareto ni Parsons ne se rallient à la thèse selon laquelle l'équilibre du système est garanti au moment où tous ses composants sont en position d'immobilité et ils y reviennent spontanément si une perturbation provient de l'extérieur. Les deux donnent à la notion des significations si différentes (entropie maximum, introduction d'un ordre diminuant l'entropie, processus autonome d'équilibration, équilibre des conditions permanentes, activités et ouvertures dans les échanges, processus fonctionnel différent de la structure, etc.) que chacune d'elles peut être conçue comme une métaphore en soi, par ailleurs difficilement comparable ou intégrable avec les autres dans un ensemble unique. Et pourtant chacune d'elles, généralisant certaines conclusions à un autre domaine, à un autre niveau d'action, éclaire bien l'objet étudié et met en évidence les imbrications des contextes et de relations. Grâce à ces relations, on arrive à organiser, avec des catégories disponibles, les phénomènes perçus par les sens et à les rendre intelligibles en première approximation ${ }^{10}$.

9 J'ai discuté ce point dans le chap. 1 de mon livre Critiques du savoir sociologique, Paris, PUF, 1993, pp. 21-45.

10 Une présentation précise de toute la problématique se trouve in J. Baechler, Le schème de l'équilibre en sociologie historique, in: Sous la direction de A. Bouvier, Pareto aujourd' hui, Paris, PUF, 1999, pp. 221-240. 
Les métaphores tirées de (2) l'organicisme sont encore plus nombreuses et chez les pères fondateurs de la sociologie se marient avec les mécaniques et elles n'ont que des liens assez lâches avec la pratique méthodique de traitement des informations empiriques. On peut même ajouter que les métaphores organiques constituent le lieu commun le plus ancien et le plus utilisé des représentations de la société. D'elles, par des procédures inférencielles hasardeuses ${ }^{11}$ et des hypothèses risquées, par des opérations indifférentes aux règles des démarches empiriques (constats, mesures, mises en relations, constructions et interprétations liées entre elles), Spencer et Durkheim ont dérivé la théorie du passage du simple au complexe; Comte, Marx, Tylor, Morgan celles des étapes ou des stades du développement; Parsons et Merton la théorie de l'évolution sociale et Smelser, avec tant d'autres, celles du progrès et du changement ${ }^{12}$.

L'organicisme a permis de concevoir la société comme un organisme biologique, en tant que système relativement fermé, ayant une finalité prépondérante, celle de survivre et croître. Pour réaliser cette finalité, la société doit s'adapter au milieu et mettre en place des stratégies complexes afin de se produire et se reproduire. Les divers types de sociétés (société de chasse et de cueillette, pastorale, horticole, agricole, industrielle, etc.) correspondent aux stades de développement de l'organisme vivant. Les changements sont engendrés par les lois naturelles du développement. Ils relèvent rarement du hasard; ils sont lents, continus, progressifs, linéaires, nécessaires, endogènes. Les sociétés primitives sont celles dont l'évolution ne fait que commencer ou qui s'est bloquée. D'où les métaphores analogiques, utilisées même par Jean Piaget, entre les enfants et les primitifs, entre les stades et périodes dans le développement de l'intelligence chez l'enfant et l'histoire des sciences, entre la psychologie génétique et l'épistémologie. Les analogies biologiques permettent d'identifier dans les sociétés primitives les facteurs de croissance des sociétés modernes, mais à une phase antérieure de leur évolution. Elles deviennent ainsi les présupposés de la plupart des théories du changement, du développement et de la modernisation.

Que la société soit un organisme dont les fonctions, par ailleurs indéfinissables en termes de conduites observables empiriquement, visent de façon téléologique à en garantir la survie, ce postulat d'universalité évolutive, transposé mécaniquement de l'organisme biologique à l'organisme social, demeure, pour le moment, très problématique: sa logique est circulaire, ses hypothèses invérifiables, ses généralisations irréfutables, ses apories nombreuses et ses contradictions internes caractérisées par d'insurmontables difficultés conceptuelles. Il n'en reste pas moins que les différentes versions de cette métaphore organique aident à circons-

11 L'usage de l'inférence en sciences sociales soulève des questions et des doutes insolubles ainsi qu'on peut le constater à la lecture du livre de R. Nisbett \& L. Ross, Human Inference : Strategies and Shortcomings of Social Judgment, Englewood Cliffs, NJ, Prentice-Hall, 1980.

12 A ces métaphores sont consacrés les chapitres 7 et 8 du livre de R. A. Nisbet, Social Change and History, New York, Oxford University Press, 1969. La sociologie weberienne du changement social n'est pas immune de la contagion organiciste ainsi que j' ai pu le constater en relisant les travaux d'un très original disciple de Weber. Voir à ce propos: G. Busino, La contribution de Reinhard Bendix à l'élaboration de la sociologie historique, in «Revue internationale de politique comparée», vol. 5, n. 3, Hiver 1998, pp. 507-520, et D. E. Apter, Bendix et la modernisation, Ibid., pp. 521-541. 
crire certains phénomènes autrement indiscernables, à réduire la complexité, et pour ces raisons elles continuent à prospérer, ainsi que les travaux d'Edward $\mathrm{O}$. Wilson et la sociologie de la «bio-cultural evolution» du sociologue Joseph Lopreato nous le prouvent.

Les sociologues font aussi appel aux métaphores économiques (3) à cause de la proximité de l'économie avec les sciences naturelles. Certains d'entre eux, fascinés par les progrès de la science économique, pensent que l'adoption de la méthode économique sortira la sociologie de l'impasse. Dès lors, ils empruntent à l'économie néoclassique son postulat fondateur: la société se compose d'individus juxtaposés, recherchant leur intérêt de manière rationnelle. Ils entrent en contact les uns avec les autres par l'intermédiaire du marché afin de maximiser leurs intérêts. Un ordre est ainsi créé grâce aux mécanismes d'ajustement automatique du marché. Les agents cherchent toujours à atteindre leur propre position d'équilibre. Chaque agent est doté de volonté-liberté et de capacité-efficacité, qualités indispensables pour réaliser ses objectifs. Les sociologues vont étendre de façon métaphorique ce paradigme du marché aux sphères non marchandes, en somme à tous les aspects de la vie sociale. Gary S. Becker affirme: «En fait, la théorie économique est peut-être en passe de fournir un cadre unifié à tout comportement qui met en œuvre des ressources rares, non marchandes, non monétaires aussi bien que monétaires, internes à un groupe restreint aussi bien que concurrentielles.» Et ailleurs, le même dit: «Tout comportement humain peut être conçu comme mettant en jeu des participants qui maximisent leur utilité à partir d'un ensemble stable de préférences et qui accumulent la quantité optimale d'informations et autres intrants sur une variété de marchés.» ${ }^{13}$

Cette métaphore économiciste implique, entre autre, la constitution de toutes les sciences sociales en économie généralisée des comportements humains, du mariage à la criminalité, de l'adultère à la justice, des associations sans buts lucratifs au marché religieux, du vote électoral à l'assistance aux pays en voie de développement. Chaque individu ne trouve le sens de ses actes qu'à maximiser ses utilités. Produire ces mêmes utilités de façon efficace reste le seul, l'ultime enjeu de ses choix qui portent sur le temps, le seul bien vraiment rare. Un exemple servira à illustre ces propos, exemple tiré de $A$ Theory of Marriage que Becker a publié en 1981. D'après cet auteur, pour analyser la famille, il faut s'en référer à la théorie économique de l'entreprise, car la famille n'est rien d'autre qu'une «petite usine ». Elle se constitue exactement comme une entreprise. En effet, le contrat de mariage est le résultat d'un processus de tâtonnement sur le marché du mariage. L'enjeu - le choix du conjoint - concrétisera la maximisation des utilités liées de deux individus. Dans la famille, les individus produisent des satisfactions finales, objets d'une fonction d'utilité familiale contribuant à transformer toute consommation en production. Il est alors facile de formaliser la production de ces utilités et de constater que la fonction de production domestique introduit le temps comme un bien rare. Dans une situation de maximisation des utilités sous certaines contraintes (une contrainte de budget et une contrainte de temps), comment les membres d'une famille vont agir? En utilisant leur temps entre la production

13 G. S. Becker, The Economic Approach to Human Behavior, Chicago, University Press, 1976, p. 205 et p. 14. 
de revenus par le travail salarié et la satisfaction de certains besoins d'origine non marchande. De tels emprunts, permettent, certes, de traiter la famille comme s'il s'agissait d'une entreprise; cependant, ils nous contraignent à considérer le temps dans la famille comme l'équivalent du temps du travail salarié, à confondre le privé avec le public, à escamoter le fait que dans le travail domestique il y a une part non mesurable, car invisible socialement, à ignorer que les échanges ne peuvent pas tous être transcrits en langage monétaire et formalisés. Derrière tout cela, il y a, bien entendu, une représentation métaphorique de la société en tant que mécanisme de régulation automatique, en tant qu'ordre naturel, en tant que système physique ou organique.

Ces emprunts métaphoriques ont donné naissance à un important courant intellectuel que l'on nomme aujourd'hui «théorie de l'action rationnelle» (Rational Action ou RAT ou encore Rational Choice). Il regroupe des tendances différentes: la «logique de l'action collective», le «rationalisme méthodologique», $l^{\prime}$ «actionnalisme» et l'«individualisme méthodologique». Tous les tenants de ce courant empruntent à l'économie néoclassique la métaphore selon laquelle les acteurs sociaux agissent en fonction de leur préférence, et donc se comportent de manière rationnelle. La tendance purement économiciste exclut, en principe, de sa rationalité la solidarité, le pouvoir et le prestige; celle du rationalisme les comportements gouvernés par des normes et des règles; celle de l'actionnalisme les croyances et les préférences; celle de l'individualisme n'importe quelle forme de totalité. Toutes ces tendances n'aboutissent point aux mêmes résultats. Par exemple, l'individualisme méthodologique peut renverser ou éliminer son économisme de départ, alors que le rationalisme économique doit rompre avec l'individualisme méthodologique pour rester fidèle à ses corollaires (agir de façon égoïste, exclusivement pour ses intérêts, se contenter de "satisficing» plutôt que de maximisation ou d'optimisation, divers facteurs affectent la fonction d'utilité, l'information est toujours correcte et l'environnement de l'action est toujours constant et donné).

La métaphore sociologistique (4) trouve en Durkheim son créateur. L'auteur de Les règles de la méthode sociologique, ainsi que François Bourricaud l'a montré de façon magistrale ${ }^{14}$, n'est jamais arrivé à s'arracher vraiment aux enchantements et aux facilités des approches mécaniste et organiciste; ni à se débarrasser de la croyance qu'il n'y a d'autre voie dans l'étude des sociétés que celle fournie par les règles, les procédures et les raisonnements déductifs et inductifs, par les modèles de rationalité des sciences physiques. Durkheim répète que les faits sociaux sont des choses, qu'ils n'ont rien à faire avec la psychologie, qu'ils sont des représentations d'un sens collectif, que la société est un super-organisme précédant et constituant les individus ainsi que leurs sentiments et leurs croyances. La conscience collective ne dépend pas ontologiquement des intentionnalités individuelles ni elle n'est la résultante des comportements individuels à expliquer. Elle est génitrice d'histoire mais entièrement étrangère à l'histoire. Ces flux d'emprunts conceptuels, ces usages métaphoriques de concepts élaborés à l'abri des astreintes spatiotemporelles, font que l'explication sociologistique se base sur des concepts réifiés,

14 F. Bourricaud, Contre le sociologisme: une critique et des propositions, in « Revue française de sociologie», XVI, suppl. 1975, pp. 583-603. 
qu'elle donne lieu à un savoir aux frontières incertaines, à l'identité nébuleuse, aux résultats cognitifs contestables, à des nombreuses contradictions internes, mais aussi à des incohérences de méthodes, comme celles que les catégories de l'anomie et du fatalisme produisent dans le cadre de l'analyse causale.

La culture et la langue (5), en tant que charpente de la société, en tant que système d'expression et de communication pour définir l'action sociale, a commencé très tôt à intriguer les sociologues. Marcel Mauss a souvent écrit que le social constitue un texte à lire, que les comportements doivent être déchiffrés en tant que rapports d'échanges, de réciprocité et d'équivalence, que les faits sociaux sont des réalités symboliques, dotées de règles grammaticales, avec des morphèmes et des phonèmes. Depuis, pour enraciner la réalité sociale dans l'univers symbolique, trois approches ont été élaborées par les sociologues: l'interactionnisme symbolique, l'ethnométhodologie et le structuralisme. En appliquant métaphoriquement à la sociologie plusieurs schémas analytiques de la linguistique générale, et notamment ceux de diachronie/synchronie, forme/substance, langue/parole, logique/langage, sens/signe, les sociologues ont cru mettre en évidence les systèmes de règles qui régissent la société, son fonctionnement ici et maintenant, découvrir le mode de production des significations ainsi que la modalité de construction de la réalité sociale. Néanmoins, ils n’ont jamais pu caractériser la spécificité de la langue par opposition aux constructions sociales qu'elle serait capable d'élaborer et de façonner. Si la langue organise et conditionne notre manière de concevoir le monde, nos comportements sociaux, nos systèmes symboliques, doit-on alors dire que l'ordre social est langue? Mais puisque ni la langue ni la société n'ont un statut ontologique transcendantal, puisque ni la linguistique générale ni la sociologie ne possèdent d'unités concrètes susceptibles d'être immédiatement reconnues, les sociologues se sont trouvés dans une impasse. Cependant, les emprunts linguistiques transformés en métaphores et en canons heuristiques ont permis à Herbert Blumer et à Erwing Goffman, à Harold Garfinkel et à Aaron Cicourel de montrer comment se mettent en place les opérations de désignation moyennant lesquelles les objets sociaux et le système social sont construits. L'analyse conversationnelle de Harvey Sacks et de E. A. Schegloff, les logiques argumentatives de Stephen Toulmin et de Jean-Blaise Grize ont ouvert de belles perspectives sur la persuasion et les logiques non démonstratives de la communication et, en général, sur les représentations connotatives du monde quotidien. De la métaphore linguistique la sociologie a tiré deux hypothèses majeures: (a) le langage parlé est un système de signes parmi d'autres; (b) tout système de signe a un caractère hermétique et auto-référentiel.

L'usage que Claude Lévi-Strauss a fait de ces deux hypothèses pour analyser les règles du mariage et les règles de parenté, ainsi que les mythologies, est bien connu. L'analyse des systèmes de signes verbaux et non verbaux a obligé les sociologues à établir des liens entre la conscience historique et les aspects normatifs de la science, à s'intéresser à l'herméneutique, à l'interprétation, à la lecture des systèmes culturels, à décoder les comportements individuels et sociaux gouvernés par des normes et des règles, les actions élaborées en fonction de modèles normatifs et de systèmes de règles et gouvernés par la cohérence qui caractérise tout modèle normatif et tout système de règles.

Cependant la métaphore linguistique, indifférente à la connaissance absolue ou exprimée en termes de probabilité statistique, pose des problèmes compliqués 
et difficiles à résoudre. Si l'objet est construit grâce au processus de désignation, si la langue définit la situation et la montre, il est alors impossible de caractériser les messages, les informations, les sensations; il est problématique de réduire la désignation de l'objet à sa représentation. Si la définition et la compréhension de la situation découlent du processus de désignation qui ne distingue pas les relations sociales en tant que langage du langage en tant que relations sociales, la connaissance reste fatalement limitée à un univers particulier et spécifique dont la clôture sémantique est inéluctable.

La métaphore théâtrale, la mise en scène, la dramaturgie (6) présument que le monde social serait une une scène et que chaque individu y tient un rôle ${ }^{15}$. Or il n'y a aucun isomorphisme entre la scène théâtrale et la vie sociale, entre les sujets sociaux et les acteurs, entre l'intrigue d'une pièce de théâtre dont le déroulement et la fin ont été agencés préalablement et qui sont donc connus à l'avance, et l'imprévisibilité de l'enroulement de l'existence sociale faite souvent d'actions aux conséquences non voulues et de réactions et résultats parfois aux effets pervers. Les expériences théâtrales sont momentanées et passagères, imaginées et ordonnées par un auteur, prédéterminées et préstructurées par lui seul; elles vivent durant l'espace d'une représentation, d'un spectacle, elles sont prévisibles et toujours à distance du spectateur, alors que les expériences sociales se font au hasard des improvisations de tout un chacun, elles sont duratives, inévitables, nous entourent de tous les cotés et leurs conséquences restent imprévisibles, non préméditées, non volontaires, non structurées. Pour cela la vie sociale ne peut même pas être comparée à une «commedia dell'arte», puisque les acteurs ici nouent et dénouent une trame donnée à l'avance, qu'ils peuvent inventer des reparties inopinées, alors que dans la première les aléas et les contraintes normatives déterminent la portée des actions, la liberté limitée de l'agent social. Toutefois la métaphore dramaturgique apporte quelques lumières lorsqu'elle est appliquée à certains domaines spécifiques de la vie sociale (cérémonies initiatiques, rites de passages, conflits de rôles, crises, etc.) ainsi que le fait avec doigté, par exemple, E. Goffman dans La mise en scène de la vie quotidienne où nous lisons: «On peut supposer que toute personne placée en présence des autres a des multiples raisons d'essayer de contrôler l'impression qu'ils reçoivent de la situation. On s'intéresse ici à certaines des circonstances le plus souvent associées à l'emploi de ces techniques. [...] Quand un acteur joue le même rôle pour un même public en différentes occasions, un rapport social est susceptible de s'instaurer.»

Dans des cas spécifiques, la métaphore théâtrale peut être isomorphe à la vie sociale et en conséquence elle peut aider à élaborer des opérations de spécifications de quelques utilités pour l'analyse.

La métaphore des jeux (7) fait des emprunts à la théorie du même nom, formulée en 1944 et depuis ayant connu diverses applications et généralisations. Sans faire ici l'historique de ces avatars ${ }^{16}$, il suffit de rappeler que la théorie des jeux étudie la prise de décision en situation de concurrence, de conflit, de la part

15 J'ai analysé le concept de «rôle» au chap. IV de mon livre La sociologie sens dessus dessous, Genève, Droz, 1992, pp. 87-104.

16 Pour une information précise, lire l'article de L. Dell'Aglio, Alcune questioni sull'evoluzione della teoria dei giochi, in PRISTEM/Storia, Note di Matematica, Storia, Cultura, a cura di P. Nastasi, Milano, Springer-Verlag Italia, 1998, pp. 33-51, avec une importante bibliographie. 
d'acteurs cherchant à satisfaire chacun ses propres intérêts. Ces derniers peuvent être convergents ou divergents selon le contexte. La théorie, afin de donner une formulation mathématique à des situations complexes et compliquées, doit procéder à des simplifications et postuler un principe de rationalité, dit principe du maximin, selon lequel un acteur tente, en évaluant les risques et les solutions avant d'arrêter le choix, de maximiser ses gains tout en sachant que son adversaire, doté de la même rationalité que lui, tentera de minimiser ses pertes.

La métaphore sociologique doit présumer un type de comportements et des formes de conduites sociales gouvernés par un système de règles dont le déterminant majeur est le calcul des coûts et des bénéfices. Qu'il y ait des actions et des situations où les choix sont marqués par une rationalité basée sur la maximisation du profit, c'est évident, mais il s'agit de cas limités, sporadiques, pour ne pas dire exceptionnels, dans la vie quotidienne. L'action sociale est déterminée par les liens sociaux, par la conscience, l'intentionnalité, les réciprocités des perspectives, en somme par des logiques naturelles et des sentiments, le tout façonné et gouverné par les applications de modèles normatifs souvent affectés d'idiosyncrasie au calcul coût/bénéfice. Si le sociologue néglige cette donnée fondamentale du problème, ou s'il prend en considération l'efficacité des moyens employés en faisant abstraction du profit recherché, l'usage de la métaphore ne produit qu'une explication incohérente et circulaire, tautologique. Norbert Elias a écrit à raison dans Qu' est-ce que la sociologie?: «Les théories sociologiques qui présentent les choses de telle manière que les normes semblent être en quelque sorte les causes des relations sociales entre les hommes, ignorant qu'il pourra exister des relations humaines non normalisées et non réglementées, donnent des sociétés humaines une image aussi fausse que les théories qui ignorent le fait que les relations humaines, originellement non normalisées et non réglementées, puissent être soumises à la normalisation.» J'ajoute que Raymond Boudon a mille fois raison de répéter inlassablement que les applications de la théorie des jeux à l'analyse sociologique sont, à ce jour, pratiquement inexistantes, que ses apports sont inapplicables à des données observées, qu'elle doit être considérée comme une doctrine spéculative susceptible de rendre compte uniquement de situations délibérément idéalisées. Sous cet angle-là, elle peut nous aider à formaliser quelques secteurs du langage des sciences sociales et ainsi à analyser certains mécanismes sociaux idéalisés ${ }^{17}$.

La métaphore du réseau (8), mise au point par Simmel, Moreno, RadcliffeBrown, Firth, Barnes ou Bott, connaît un développement important depuis les années '70, mais aussi des variations sémantiques à tel point divergentes qu'il est difficile de les présenter ici. Le sociologue dit que le réseau est l'ensemble des relations existantes (ayant toutes les attributs de centralité, cohésion et équivalence de positions) entre l'ensemble des acteurs ou des éléments. Ces relations sont de nature disparate. Un réseau peut être organisé ou pas. Pour le besoin de l'analyse, il faut en tracer les frontières et présumer qu'il constitue une totalité dont les éléments ont des degrés d'interdépendance. Dès lors sa structure ne peut être saisie qu'après coup et décrite avec des graphes. Elle ne préexiste pas aux relations, elle émerge des interactions.

17 Des bons exemples peuvent se trouver dans le recueil édité par P. Hedström et R. Swedberg, Social Mechanisms. An Analytical Approach to Social Theory, Cambridge, University Press, 1998. 
Les atouts de cette métaphore sont évidents pour élaborer des hypothèses très riches (les structures émergent des relations et des interactions et puis elles contraignent les comportements), à condition toutefois que l'analyste présuppose que l'acteur agit de façon rationnelle s'il pense à son avantage et qu'il fait des choix seulement après avoir comparé les avantages et les inconvénients. Nous rencontrons ici les mêmes difficultés que la théorie des choix et des anticipations rationnels a laissées en suspens. Pour les autres utilisations de cette métaphore, il suffit de renvoyer aux travaux de Michel Callon, de Bruno Latour et de Manuel Castells ${ }^{18}$.

Il n'y a pas de critères pour comparer entre elles les métaphores usitées en sciences sociales ni nous n'avons de tests empiriques pour pondérer leur pertinence. L'analogie fonctionnelle et l'homologie sont rares car la similarité caractérisant des objets semblables est normalement indéfectible. L'isomorphisme de forme ou de structure entre deux systèmes à contenus différents n'est presque jamais réalisée. Et pourtant les métaphores nous permettent de «parler» de phénomènes qui se dérobent à une transcription directe; elles donnent de la vie à des nouveaux objets d'expérience, à des nouveaux champs perceptifs, voire à des nouveaux langages. Grâce à cela, nous pouvons effectuer avantageusement, rapidement, économiquement des opérations d'élaboration, de spécification et de formalisation moyennant lesquelles la construction de modèles matériels, formels mathématiques, formels qualitatifs ou idéal-types, expérimentaux, simulés, sont relativement aisées. Ces modèles, dérivés des métaphores mais transformées quand cela est possible - en métaphores élaborées et déterminées, facilitent la réflexion théorique, l'évaluation des cohérences internes, l'adéquation avec l'objet, le dégagement de toutes les conséquences possibles.

Certes, les modèles étant irréels ne permettent pas de relever des correspondances entre une mesure et la réalité sociale, mais ils fournissent une aide puissante pour établir l'indispensable rapport logique entre les définitions nominales et les définitions opératoires. Artifices illustratifs, les modèles, bien qu'ils produisent des savoirs non cumulatifs, permettent d'éliminer les ambiguïtés et les équivoques du langage et ainsi de mieux interpréter et expliquer les phénomènes sociaux, de produire des effets d'intelligibilité.

Le langage des sciences sociales, et notamment de la sociologie, est agencé par une série de métaphores, lesquelles ont des liens très ténus avec la matrice originelle. Ce langage présente les deux structures argumentatives déjà décrites par Aristote: il va du fait à la règle, du fait au fait, du particulier au général (l'induction, l'exemple), et du général au particulier (la déduction et l'enthymème dont les prémisses probables peuvent rester implicites).

Les métaphores de base et les analogies établissent, implicitement ou explicitement, les prémisses communes, l'accord préalable sur les faits, les vérités, les présomptions, entre tous les membres d'une même communauté scientifique. Elles permettent de donner une assise aux arguments quasi logiques (arguments qui s'apparentent à l'identité et à la transitivité mais qu'on peut réfuter), à ceux

18 Pour les travaux de Callon et Latour, voir: G. Busino, Sociologies des sciences et des techniques, Paris, PUF, 1998. Pour les travaux du sociologue espagnol, il suffit de renvoyer à son livre $L a$ société en réseaux. L'ère de l'information, Paris, Fayard, 1998. Voir aussi A. Muti, Reti sociali: tra metafore e programmi teorici, «Rassegna italiana di Sociologia», 1996. 
s'appuyant sur l'expérience, à ceux fondant la structure du réel, à ceux procédant par dissociation de notion.

C'est ainsi que les métaphores permettent la fabrication du raisonnement sociologique structuré avant tout par la logique de l'argumentation au sens que cette expression a reçu de Jean-Blaise Grize ${ }^{19}$. Pour cette raison, je peux faire miennes les conclusions de Jean-Claude Passeron, selon lequel: «1. Les sciences empiriques sont des langages de description du monde qui doivent produire un type particulier de connaissance aux épreuves empiriques que la structure logique de ces langages rend possibles et nécessaires. 2. Il n'existe pas et il ne peut exister de 'langage protocolaire' unifié de la description empirique du monde historique. 3. La mise à l'épreuve empirique d'une proposition théorique ne peut jamais revêtir en sociologie la forme logique de la 'réfutation' ('falsification') au sens poppérien.» ${ }^{20}$

Le chantier des métaphores et des analogies est un chantier stratégique pour l'épistémologie des sciences sociales. Il a besoin des collaborations des spécialistes de tous les domaines des sciences de l'homme et de la société. Les articles réunis ici prouvent combien cette collaboration est indispensable pour que dans le chantier nous puissions ouvrir des portes et des fenêtres.

Institut d' anthropologie et de sociologie

Université de Lausanne

19 J.-B. Grize, De la logique à l' argumentation. Préface de G. Busino, Genève, Droz, 1982. Voir également G. Busino, Questions actuelles de sociologie de la science, Lausanne, IASUL, 1995, pp. 119-129.

20 J.-C. Passeron, Le raisonnement sociologique. L'espace non-poppérien du raisonnement naturel, Paris, Nathan, 1991, p. 359. 